\title{
Circuit and Coil Design for In-Vitro Magnetic Neural Stimulation Systems
}

\author{
Eric Basham, Student Member, IEEE, Zhi Yang, and Wentai Liu, Senior Member, IEEE
}

\begin{abstract}
Magnetic stimulation of neural tissue is an attractive technology because neural excitation may be affected without requiring implantation of electrodes. Pulsed discharge circuits are typically implemented for clinical magnetic stimulation systems. However, pulsed discharge systems can confound in-vitro experimentation. As an alternative to pulsed discharge circuits, we present a circuit to deliver asymmetric current pulses for generation of the magnetic field. We scaled the system down by using ferrite cores for the excitation coil. The scaled system allows observation using electrophysiological techniques and preparations not commonly used for investigation of magnetic stimulation. The design was refined using a comprehensive set of design equations. Circuit modeling and simulation demonstrate that the proposed system is effective for stimulating neural tissue with electric-field gradients generated by time-varying magnetic fields. System performance is verified through electrical test.
\end{abstract}

Index Terms-Circuit design, coil design, ferrite core, functional magnetic stimulation, magnetic stimulation, rate of closure, transcranial magnetic stimulation (TMS).

\section{INTRODUCTION}

$\mathbf{M}$ AGNETIC stimulation of neural tissue is an intriguing technology because stimulation may be affected without direct contact to the tissue under study. As such, magnetic stimulation offers advantages in biocompatibility, bioresistance, and operational biotoxicity in comparison to electrical stimulation. The stimulating coil may be sealed and isolated from the target tissue during magnetic stimulation. Since there is no metal-electrolyte interface, as is the case with electrodes used for electrical stimulation, issues of charge transfer, electrode surface modification, and corrosion are mitigated. In addition, magnetic fields penetrate without being attenuated through nonconductive tissue because their permeability at low frequencies $(<50 \mathrm{kHz})$ is near unity.

Magnetic stimulation may be used to stimulate neural tissue without requiring surgery to implant electrodes when applied

Manuscript received July 12, 2008; revised January 21, 2009. Current version published September 25, 2009. This work was supported in part by the National Science Foundation Biomimetic MicroElectronic Systems Research Center under Agreement No. H31512, Amendment 6, and in part by National Semiconductor, Santa Clara, CA. This paper was recommended by Associate Editor R. Genov.

E. Basham and Z. Yang are with the Electrical Engineering Department, University of California, Santa Cruz, Santa Cruz, CA 95064 USA (e-mail: ebasham@soe.ucsc.edu, yangzhi@soe.ucsc.edu).

W. Liu is with the Electrical Engineering Department, University of California, Santa Cruz, Santa Cruz, CA 95064 USA. He is also with the National Chiao Tung University, Hsinchu, Taiwan 300 (e-mail: wentai@soe.ucsc.edu).

Color versions of one or more of the figures in this paper are available online at http://ieeexplore.ieee.org.

Digital Object Identifier 10.1109/TBCAS.2009.2024927 externally. One of the most important applications of external excitation of neural tissue is transcranial magnetic stimulation (TMS). There is a wide array of literature on TMS brain mapping and neuromodulation. External magnetic stimulation has also been used as an alternative to functional electrical stimulation (FES) to mitigate incontinence [1], pain treatment [2], evaluate spinal function, and as a diagnostic tool for the evaluation of nerve damage [3]. Reviews of magnetic stimulation and TMS, in particular, are available in [4]-[9].

Magnetic stimulation of neural tissue is typically accomplished with air-core coils (centimeters in diameter) remote from the site of stimulation (centimeters distant). Spatially varying electric fields are generated by using a single damped sinusoidal current pulse [10]. The use of air-core coils, the distance from the site of stimulation, and the driver circuit topology compound to make the energy requirements for magnetic stimulation significant (i.e., in the range of Joules). Magnetic stimulation with traditional methods confounds in-vitro experimentation as the area of effect is quite large and interferes with standard electrophysiology recording equipment. The resulting electromagnetic interference (EMI) and mechanical constraints are especially evident in adherent cell electrophysiological studies. Typically, only large and long neural preparations (centimeters in length) can be used for experimentation.

While extensive magnetic stimulation modeling work has been presented [11]-[16], considerably less quantitative in-vitro work has been performed [17], [18]. In-vitro experiments are critical for characterizing the site of action, the structures stimulated, and the long-term tissue histological effects of magnetic stimulation. A properly scaled system also facilitates the study of the histological effects of magnetic stimulation and aids the investigation of pulsed electromagnetic (EM) fields on nerve regrowth. These are both active areas of investigation [19]-[21]. In-vitro studies can be particularly useful for studies of localized gene regulation and expression due to magnetic stimulation. An in-vitro system enables precise control and isolation of experimental variables.

While electrical fields from arrays of electrodes can approximate magnetic stimulation, in-vitro systems can provide critical insight into complex, often contradictory results common in magnetic stimulation experiments. For example, in the stimulation of one-dimensional (patterned) neuronal cultures, cultures displayed similar morphology, electrical response, and spontaneous activity but the response to magnetic stimulation was successful in only $22 \%$ of all cultures and $64 \%$ of similarly patterned cultures [22]. The authors posited the existence of "initiating neurons" with a lower magnetic stimulation threshold. In this paper, the difficulty in aligning morphological features 


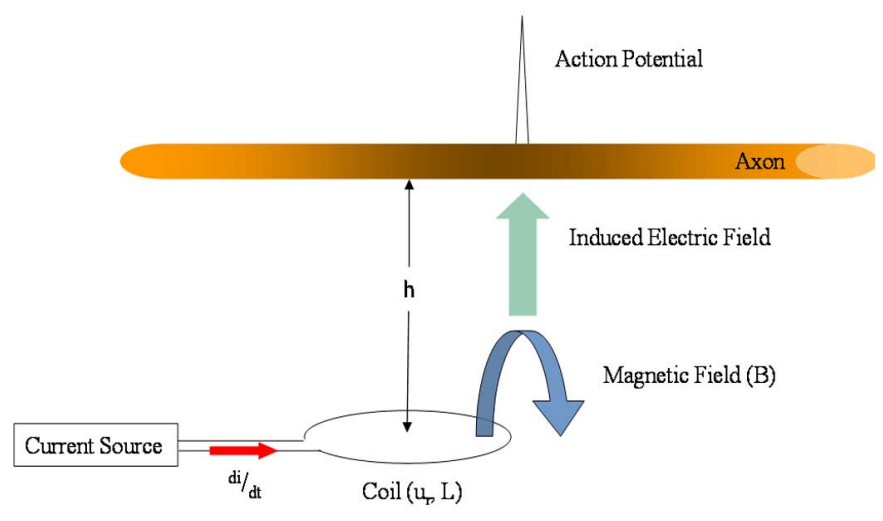

Fig. 1. Magnetic stimulation system diagram. B is the magnetic field, $u_{r}$ is the coil relative permeability, di/dt is the current $\operatorname{ramp}, L$ is the coil inductance, and $h$ is the height above the coil. These terms represent the design variables for an in-vitro magnetic stimulation system. The shaded area on the axon represents the depolarized area of the fiber.

to coil activation areas is specifically mentioned. Small-scale coils and flexible stimulation systems meet this need. Similar cases may be found in clinical experiments as well. In a recent clinical evaluation of repetitive TMS (rTMS), the key difference in efficacy was found to link closely with the manufacturer model (and, thus, the specific waveform) generating the biphasic pulse [23]. Modifying full-scale magnetic stimulation systems in a clinical environment presents significant challenges to proof-of-concept experimentation. As an example, there is little data that covers co-stimulation (pairing electrical stimulus with magnetic stimulus) and paired pulse protocols. Rapidly reconfigurable in-vitro systems can be invaluable for developing better predictive models, correlating effects of clinical experiments [24], and developing proof-of-concept systems.

Selective stimulation, recruitment studies, and nerve impulse blocking experiments are excellent examples of the need for more flexible systems. For blocking experiments, the blocking pulse must last until the action potential has propagated from the site of initiation. In full-scale systems with large coils, it is challenging to create pulses of the necessary amplitude and duration, given the use of large coils and the distance between stimulation sites [25]. Selective stimulation is studied with the use of subthreshold hyperpolarizing and depolarizing pulses. Full-scale magnetic stimulation systems cannot deliver variable amplitude pulses on the necessary timescales. This is especially the case when the pulsewidths are of widely different durations as the pulsewidth in full-scale magnetic stimulation systems is set by the capacitance bank and the coil inductance, as discussed in Section II. Due to the currents and voltages involved, dynamic reconfiguration of the capacitor bank and coil inductance is problematic. Much can be learned in-vitro before committing to building a full-scale magnetic stimulation system.

We have reduced the scale of magnetic stimulating systems to address the need for flexible, in-vitro systems. Scaling was accomplished by using ferrite cores to reduce the area of stimulation, increase the magnetic flux through the area of interest, and ultimately reduce the power required for stimulation. Miniaturization of the system permits the location of the neural tissue closer to the coil, mitigating the need for high-power switching devices and further reducing the overall amount of energy required for stimulation. With this approach, smaller neural preparations, such as retinal sections, brain slices, and short sections of homogenous nerve fibers are available to the experimenter. The reduced energy requirements have the advantage that more flexible circuit topologies may be employed. The current amplifier presented is capable of delivering arbitrary current waveforms into a low-resistance inductive load (the magnetic stimulation coil) while maintaining stability.

\section{APPROACH}

To determine a baseline for the circuit requirements of an invitro magnetic stimulation system, the passive cable model of axons is employed. The spatial rate of change of the electric field along the length of the axon $\partial E_{x} / \partial x$ has been shown to predict extracellular neural electrical stimulation (see the review in [26]). An equivalent expression for magnetic stimulation is [14]

$$
\lambda_{m}^{2} \frac{\partial \vec{E}_{x}(x, t)}{\partial x}=-\lambda_{m}^{2} \frac{\partial^{2} V_{m}(x, t)}{\partial x^{2}}+\tau \frac{\partial V_{m}(x, t)}{\partial t}+V_{m}(x, t)
$$

where the length and time constants of the neural membrane ( $\lambda_{m}$ and $\tau_{m}$, respectively) are defined as

$$
\lambda_{m} \sqrt{\frac{r_{m}}{r_{i}}} \text { and } \tau_{m}=c_{m} r_{m}
$$

where $x$ is the distance along the axis of the nerve fiber when the nerve fiber is aligned to the $x$ axis, $E_{x}$ is the electric field along the $x$ axis, $V_{m}$ is the transmembrane voltage defined as the voltage difference between the intracellular and extracellular fluid $\left(V_{m}=V_{\text {intracellular }}-V_{\text {extracellualr }}\right), r_{m}$ is membrane resistance times unit length $\left(\mathrm{k} \Omega \cdot \mathrm{cm}\right.$ axon length), $r_{i}$ is intracellular resistance $\left(\Omega \cdot \mathrm{cm}^{-1}\right)$, and $c_{m}$ is the membrane capacitance per unit length $\left(\mathrm{F} \cdot \mathrm{cm}^{-1}\right)$. Setting $V_{m}$ to zero leads to the definition of the "activating function," $\lambda^{2} \cdot \partial E_{x} / \partial x$, which is useful for determining the initial change in $V_{m}$.

The activating function allows estimation of stimulation based upon the membrane length constant and the spatially varying electric field [26]. There is some debate in the literature as to whether the activation function is more conveniently defined as $\partial E_{x} / \partial x$ or as $\lambda^{2} \cdot \partial E_{x} / \partial x$ [27], but here we use the activating function to refer to $\lambda^{2} \cdot \partial E_{x} / \partial x$, and define the spatially varying electric field as $\delta E_{x} / \delta x$, that is, the rate of change of the electric field with respect to the $x$ axis.

Magnetic generation of the activating function is accomplished by stimulating a coil with a time-varying current (di/dt). The current, in turn, generates a time-varying magnetic field which then generates an electric field according to (3) [28]

$$
d \vec{E}^{p}=\frac{-\mu_{o} N\left(\frac{\mathrm{d} \mathbf{i}}{\mathrm{dt}}\right) d \vec{l}}{4 \pi R}
$$

where $d \vec{E}^{p}$ is the primary electric field, $\mu_{o}$ is the permeability of free space, di/dt is the rate of change of the electric current, $d \vec{l}$ is an element of the coil, $N$ is the number or turns, and $R$ is the distance between the coil element and the point where the electric field is calculated. 
a.

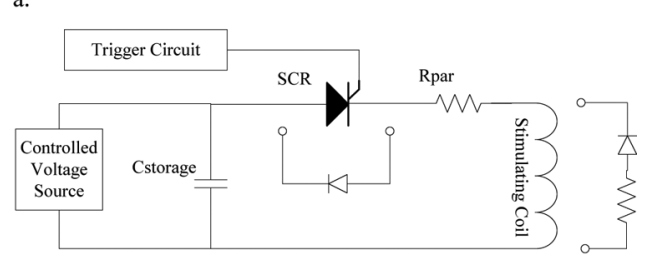

b.

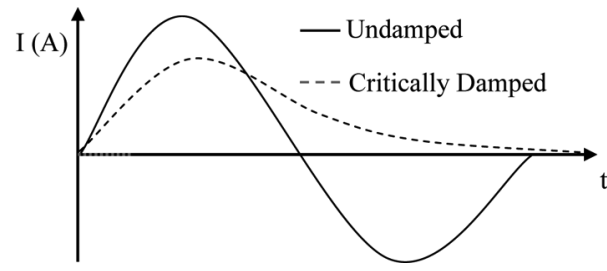

Fig. 2. Typical circuit implementation of a magnetic stimulation circuit. (a) A silicon-controlled rectifier (SCR)-triggered pulse discharge circuit implementation of a time-varying current source as in Fig. 1. (b) Resulting current waveform output. The system produces different current waveforms and, thus, different electric fields with varying shape; pulse width and magnitude as a function of the parasitic resistance in the system (Rpar); and the storage capacitance (Cstorage) and the inductance of the stimulating coil. (a) Represents a simplified implementation of a pulse discharge circuit for magnetic stimulation systems. In typical applications, Rpar is negligible and a critically damped waveform is the result of a diode and resistance in parallel with the stimulating coil as shown. In undamped applications, a diode is placed in antiparallel with the trigger circuit element-here shown as an SCR. The controlled voltage source must be current limited and, thus, appears more as a current source until the target capacitor storage voltage is reached.

The spatial electric field also varies because the magnetic field is not uniform in space. Neural structures can be placed such that the activating function is at a maximum. A conceptual diagram is shown in Fig. 1.

The more linear the current ramp is, the more uniform the electric field is during the time course of the pulse since the induced electric field is a function of di/dt. In clinical functional magnetic stimulation experiments, a constant current ramp has been shown to have lower stimulation thresholds than the commonly used damped sinusoidal waveform [29]. Modeling of the electrical stimulation pulse shape shows that the waveform shape impacts the strength-duration response and, thus, the energy required for stimulation [30], [31]. Control over waveform shape also enables the evaluation of pulse-shape-mediated nerve recruitment [32], [33]. Clearly, the ability to test the effects of waveform shape on stimulation threshold is a necessary component of a quantitative magnetic stimulation system.

Maintaining a current ramp for the length of time required to stimulate neural tissue can confound the design of a current amplifier output stage. Depending on the $\tau_{m}$ and $\lambda_{m}$ of the tissue under study, stimulation times range from hundreds of microseconds to a few milliseconds. The common strategy is to use thyristor-triggered pulse discharge circuits, as in Fig. 2. The tuned inductance-capacitance-resistance (LCR) pulse discharge circuit is used to produce a waveform with a damped sinusoidal pulse. The pulse shape $(I(t))$ is a function of the stimulating coil, the capacitor bank, and the parasitic resistance of the system according to

$$
I(t)=\frac{V}{\beta L} e^{-\alpha t} \sin \beta t
$$

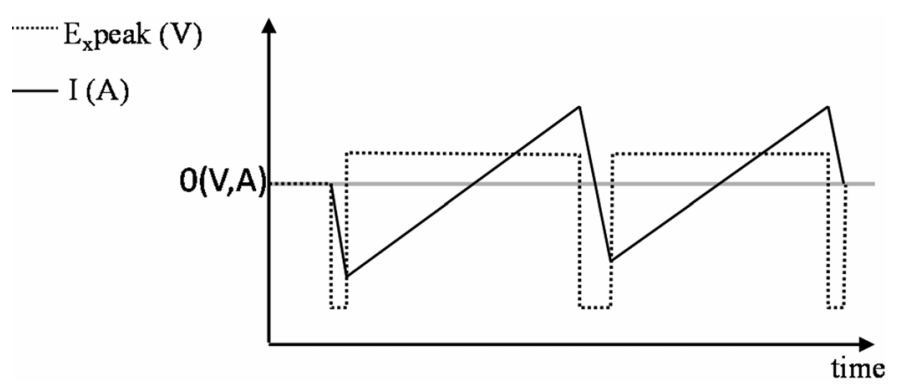

Fig. 3. Sawtooth current waveform and the resulting asymmetric biphasic electric fields produced by coil excitation. The dotted line is the induced E field, the solid line in the current waveform through the inductor.

where $\alpha$ and $\beta$ are defined as

$$
\beta=\sqrt{\frac{1}{L C}-\frac{R^{2}}{4 L^{2}}} \text { and } \alpha=\frac{R}{2 L}
$$

and $R, L$, and $C$ refer to the circuit values shown in Fig. 2(a). Shorter pulses have been shown to be more energy efficient in pulse discharge circuits [34] while the most energy-efficient time point to electrically stimulate neural tissue is at the chronaxy of the nerve [35]. Due to losses in the stimulation coil and restrictions on the circuit design window, pulse discharge circuits often operate at much shorter timescales than the chronaxy of the nerve under study.

Notable exceptions to the pulsed discharge circuit topology are the truncated pulse discharge system presented in [36] and the H-bridge type topology in [37]. In the first system, a highpower insulated-gate bipolar transistor (IGBT) is employed to stop the current as it is discharging through the magnetic stimulation coil, providing several pulsewidth measurement points. In the second system, power transistors are employed to control the linearity of the rise time and fall time of the current waveform through the magnetic stimulation coil by switching rapidly on and off during the current rise and fall times. The system presented in [36] is a particularly detailed example of a magnetic stimulation system. Another detailed example is presented in [38] and design methods are presented in [10] and [39].

In all of these cases, however, the waveform duration is a function of the capacitance storage bank size and the load inductance. Small coils, as would be employed in in-vitro systems or magnetic stimulation systems using arrays of smaller coils [40], [41] present a particular challenge when using pulsed discharge systems.

Often, they may have inductances that are much smaller than coils used in full-scale magnetic stimulation systems. As seen in (4), pulsewidth varies as a function of the coil inductance. Often, the parasitic resistance is nonnegligible because of the wire diameter, number of turns, and the space constraints of an in-vitro system. The dependence on circuit parameters of pulse discharge circuits also complicates direct comparison between coils during experimentation.

An alternative approach is to use a sawtooth to generate several concurrent current ramps as in Fig. 3. The asymmetric sawtooth (long rise time, short fall time) also allows a significant reduction in the power dissipated in the inductor as $P=I^{2} R$, provided the waveform has no direct-current (dc) component. 
The current supplied to the load is a function of the inductance of the coil when using pulse discharge circuits. In contrast, the current supplied to the load with an asymmetric current source is independent of the load. Decoupling the load from the system performance provides the opportunity to test different inductances without having to redesign the stimulus system or make it overly complex to support a wide experimental range. Feedback ensures linearity.

Using the sawtooth current driver approach also decouples the induced waveform from the coil electrical properties [42], allowing easier investigation of waveshape variation. It is easy to change the polarity of the activating function by changing the input waveform to allow direct comparison of polarity effects. A series of pulses also opens up the opportunity to test magnetic stimulation effects at longer durations than in the typical pulse discharge circuit. Charge accumulation by using closely spaced pulses is discussed further in [43]. The current driver approach requires reducing the peak current and peak voltage requirements for magnetic stimulation to the point that the output stage can be designed to deliver a repetitive sawtooth current waveform with sharp transitions. A logical approach to reduce the current requirements for the output stage is to increase the flux in the inductor loop.

A core is a very effective way to increase the magnetic flux. There are some examples in the literature of ferrite and iron cores used for magnetic stimulation [44]-[47]; however, the use of cores with a pulsed sawtooth current waveform has not been reported. Cores function essentially as flux concentrators and electric field is related to flux density as described by Faraday's law

$$
\nabla \times \vec{E}=-\frac{\partial \vec{B}}{\partial t}
$$

where $\nabla \times \vec{E}$ indicates the curl of the electric field and $\vec{B}$ is the magnetic field. The inclusion of a core is analogous to increasing the number of windings and the magnetic field capable of generating an electric field

$$
B \propto \mu_{r} \mu_{0} n I
$$

where $\mu_{r}$ is the relative permeability of a core in a solenoid, $n$ is the number of turns per unit length, or "turns density," and $I$ is the current. The core becomes a gapped inductor with an equivalent distributed permeability $\mu_{e q}$

$$
\mu_{e q}=\frac{\mu_{r}}{1+\mu_{r} \frac{l_{g}}{M P L}}
$$

where $\mu_{r}$ is the relative permeability of the core, $l_{g}$ is distance the magnetic flux travels through the air gap (gap length), and MPL is the total length of the flux path [48]-[50].

\section{Modeling AND SimUlation}

\section{A. Analytical Calculation of the Spatial Electric Field}

Analytical expressions have been derived for estimating the spatial change of the electric field $\partial E_{x} / \partial x$ as a function of coil dimensions, input current waveform (di/dt), and distance from the plane of the coil. The approach follows the strategy outlined in [28]. The resulting expression is then modified by the equivalent permeability (8) calculated for the core/winding geometry. The modeling and design effort focused on square and quad square coils because they have been shown to generate a larger activating function per unit current than other topologies [51]. Square and quad square cores also facilitate the precise alignment of neural structures because the negative and positive peak $\partial E_{x} / \partial x$ occurs at the corners of the coil. For the square coil, the spatially varying electric field component of the activating function is

$$
\frac{\partial E_{x}}{\partial x}=\frac{\mu_{e q} N}{4 \pi}\left(\frac{\mathrm{di}}{\mathrm{dt}}\right)(a+b+c-d)
$$

where $a, b$, and $c, d$ are given by

$$
\begin{aligned}
a & =\frac{1}{\sqrt{(s-x)^{2}+(s-y)^{2}+h^{2}}} \\
b & =\frac{1}{\sqrt{x^{2}+(s-y)^{2}+h^{2}}} \\
c & =\frac{1}{\sqrt{(s-x)^{2}+y^{2}+h^{2}}} \\
d & =\frac{1}{\sqrt{x^{2}+y^{2}+h^{2}}} .
\end{aligned}
$$

For the quad square core

$$
\frac{\partial E_{x}}{\partial x}=\frac{\mu_{e q} N}{4 \pi}\left(\frac{\mathrm{di}}{d t}\right)(2 a+2 b-c-d-4 e)
$$

where $a, b, c, d$, and $e$ are defined by

$$
\begin{aligned}
a= & \frac{1+\frac{x}{\sqrt{x^{2}+(y-s)^{2}+h^{2}}}}{x+\sqrt{x^{2}+(y-s)^{2}+h^{2}}} \\
& +\frac{1+\frac{x}{\sqrt{x^{2}+(y+s)^{2}+h^{2}}}}{x+\sqrt{x^{2}+(y+s)^{2}+h^{2}}} \\
b= & \frac{1+\frac{x+s}{\sqrt{(x+s)^{2}+y^{2}+h^{2}}}}{x+s+\sqrt{(x+s)^{2}+(y)^{2}+h^{2}}} \\
c= & \frac{1+\frac{x-s}{\sqrt{(x-s)^{2}+y^{2}+h^{2}}}}{x+s+\sqrt{(x-s)^{2}+y^{2}+h^{2}}} \\
& +\frac{1+\frac{x+s}{\sqrt{(x+s)^{2}+(y+s)^{2}+h^{2}}}}{x+\sqrt{(x+s)^{2}+(y+s)^{2}+h^{2}}} \\
d= & \frac{1+\frac{x+a}{\sqrt{(x+s)^{2}+(y-s)^{2}+h^{2}}}}{x-s+\sqrt{(x+s)^{2}+(y-s)^{2}+h^{2}}} \\
& +\frac{1+\frac{x-s}{\sqrt{(x-s)^{2}+(y-s)^{2}+h^{2}}}}{x-s+\sqrt{(x)^{2}+(y-s)^{2}+h^{2}}} \\
& \frac{1+\frac{x-s}{\sqrt{(x-s)^{2}+(y+s)^{2}+h^{2}}}}{x+\sqrt{x^{2}+y^{2}+(y+s)^{2}+h^{2}}}
\end{aligned}
$$


where $s$ is the side length of the square, $x$ and $y$ are the coordinates of the point in the plane of the coil, and $h$ is the height above the coil (as depicted in Fig. 1). $\mu_{e q}$ is the equivalent permeability estimate defined in (5), $N$ is the number of coil turns, and $\mathrm{di} / \mathrm{dt}$ is the ramp rate of the current source.

The analytical equations for square (9) and quad square (11) coils allow quick estimation of the spatially varying electricfield component of the activating function at any point by using a variety of core dimensions and core types. Two important details come to light using this approach. From (9) and (11), clearly field focality improves as $h$ is reduced. The stimulus efficiency of different combinations of stimulation depth to coil diameter can be evaluated with the closed-form solutions. Setting the $x$ to 0 (the peak $\delta E_{e} / \delta x$ field), $y=0$, and making the substitution with the unitless term $\zeta$, which defines the ratio between coilside length and depth of stimulation [52] as

$$
s=\frac{h}{\varsigma} \text {. }
$$

Equation (25) reduces to

$$
\frac{\partial \vec{E}_{x}}{\partial x}=-\frac{\mu_{0} N\left(\frac{\mathrm{di}}{\mathrm{dt}}\right)}{4 \pi \cdot h} \cdot\left[1-\frac{2}{\sqrt{\frac{1}{\varsigma^{2}}+1}}+\frac{1}{\sqrt{\frac{2}{\varsigma^{2}}+1}} .\right.
$$

Similar equations may be derived for double square coils and quad square (or butterfly) coils, and show that additional windings at the locus of excitation multiply the effect by either 2 (double square coils) or 4 (quad square coils). The energy required for magnetic stimulation of nerves increases dramatically as the coil is moved further away from the tissue. The area of effect also increases, leading to a less-focused area of stimulation. A similar approach is used to analyze quad coil cores with variable intercoil spacing [53].

This will allow the interaction of the system with precise structures in the neural tissue under study. Second, the current requirements to generate an activating function of the correct magnitude fall significantly with the inclusion of a core. Comparable in-vitro magnetic stimulation experiments employ di/dt ramps greater than $10 \mathrm{~A} / \mu \mathrm{s}$. By reducing the size of the coil and moving the site of stimulation closer to the coil, the current ramp requirement can be reduced to $\sim 0.1 \mathrm{~A} / \mu \mathrm{s}$. Thus, for in-vitro experimentation, an improvement in field focality and a significant reduction in energy required are obtained by moving the tissue under study closer to the stimulating coils. Scaling can reduce the maximum current peak and maximum voltage peak required to induce excitation. The reduced voltage and current requirements allow the design of more flexible current sources. The flexibility in the current-source specification, in turn, allows the investigation of waveform-based recruitment studies.

The analogous expression for point-source electrical stimulation of a fiber is

$$
\frac{\partial \vec{E}_{e}}{\partial x}=\frac{I_{e}\left(2 x^{2}-h^{2}\right)}{4 \pi \sigma\left(x^{2}+h^{2}\right)^{5 / 2}}
$$

where $\vec{E}_{e}$ is the electric field from the electrode, along the $x$ axis, $I_{e}$ is the electrode current, and $\sigma$ is the extracellular solution conductivity and $h$ is the distance from the electrode to the

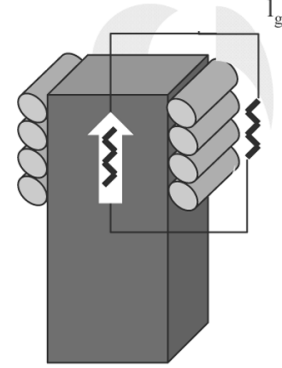

(a)

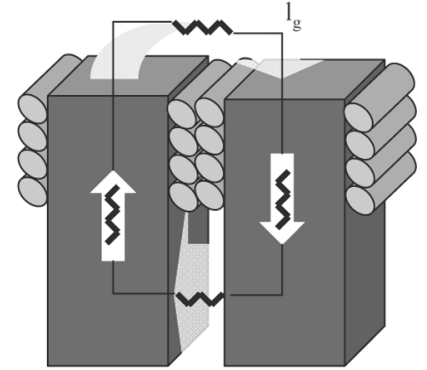

(b)
Fig. 4. Curved arrows represent the gap length (i.e., the reluctance of the air) The coil on the left is a model of a square solenoid, while the coil on the right is a model of half a quad square coil with cores.

nerve fiber. Observing the form of (14) and (15), it is seen that the spatial variation of the electric field during electric stimulation does not decrease in a similar manner to the spatial variation of the electric field generated by a magnetic field. In addition, very short pulses may exceed the charge density capabilities of microelectrodes and introduce additional variables into in-vitro experiments. Electrical stimulation with passive cable modeling provides an analogy useful for control experiments, but subtleties in the distribution of field may prove significant, especially in the light that these are first-order derivations useful for design insight.

To verify charge accumulation and define circuit design requirements, simulation was performed by using a freely available active cable model simulation tool. Neurocal is a simplified package written in MATLAB that is very easy to use and modify [54]. Testing was performed using unmyelinated nerve parameters from P. Clarkii [55], [56] and myelinated nerve parameters [57]. A typical result is shown in Fig. 5.

\section{B. Circuit Design}

A current ramp of $\sim 0.1 \mathrm{~A} / \mu \mathrm{s}$ is well within the range of a power-amplifier topology. It is critical that the rising edge of the waveform be as short as possible to prevent hyperpolarizing effects on the neural membrane, or worse inactivating the $\mathrm{Na}^{+}$ ion channels, increasing the energy requirements for stimulation. Typically a grounded load V-I converter, such as an improved Howland VCCS [58], would be used in this application. However, for a typical Howland current source as the signal frequency increases, the output impedance falls [59].

The more appropriate approach is to include the reactive load inside the feedback loop. The floating load current-source topology (Fig. 6) is particularly applicable to in-vitro magnetic stimulation as the coil is always isolated from the preparation. Both terminals of the inductor are available and it may be placed into the feedback loop and compensated. The floating load-current source is insensitive to component tolerance mismatch which plagues the improved Howland current source. In Fig. 6, $R_{s}$ functions as a sense resistor and, thus, the current across the load $\left(I_{\text {load }}\right)$ is defined as

$$
I_{\text {load }}=-\frac{V_{i n}}{R_{s}} \frac{R_{f}}{R_{i}},
$$

where $V_{\text {in }}$ is the input voltage, and the resistance are defined in Fig. 6. Equation (16) remains valid as long as $R_{f} \gg R_{s}$. 


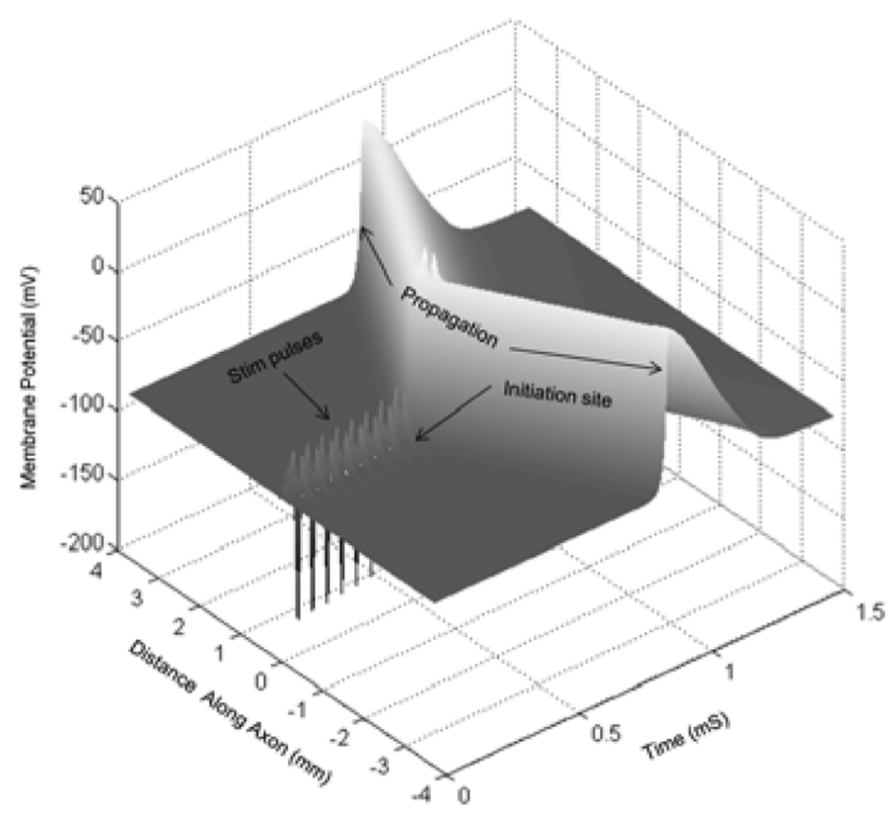

Fig. 5. Typical simulation result by using a pulsed electric-field gradient to excite an axon fiber. Ten pulses, $50-\mu \mathrm{S}$ in duration, separated by $5-\mu \mathrm{S}$ deadtimes were applied (stim pulses). The action potential is seen to initiate at the site of the maximum electric-field gradient after the ninth pulse (initiation site) and propagate along the axon in both directions from the site of initiation (propagation).

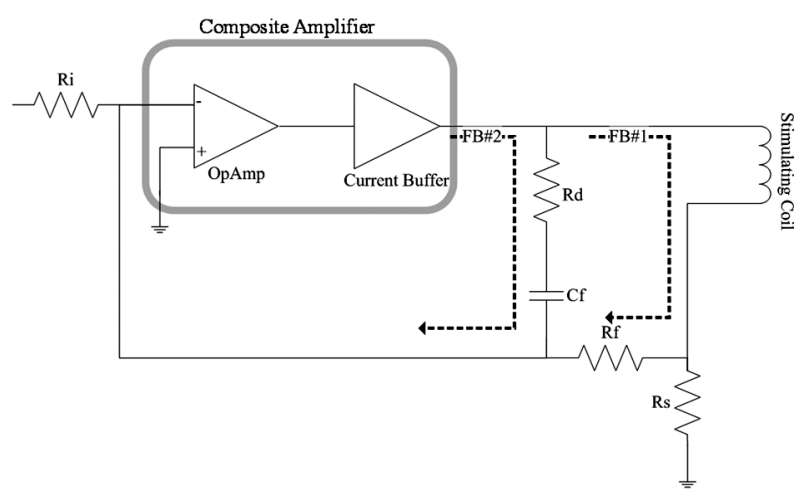

Fig. 6. Floating load-current source topology. The operational amplifier (OpAmp) and the current buffer function as a composite amplifier. Feedback paths (FB\#1 and FB\#2) are shown graphically in a bode magnitude plot in Fig. 7.

\section{Rate of Closure Stability Analysis}

The method used to analyze this circuit uses the rate of closure feedback loop analysis [60]. The open-loop gain of the amplifier is plotted. The feedback factor $\beta=V_{f b} / V_{\text {out }}$ of each independent feedback path is calculated and then the inverse $(1 / \beta)$ is plotted. The closed-loop gain follows the lowest feedback path, as in Fig. 7 .

For the case of the floating load current source, the dc-loop response is calculated when the inductor is shorted as

$$
\begin{aligned}
V_{f b}=V_{\text {out }} \frac{R_{i}}{R_{f}+R_{i}} & \quad R_{s}\left(R_{f}+R_{i}\right) \\
& \cdot \frac{\left.\left.R_{f} R_{s}+R_{l}+R_{s}\right)+R_{l}+R_{s}\right)}{R_{l} R_{l}} .
\end{aligned}
$$

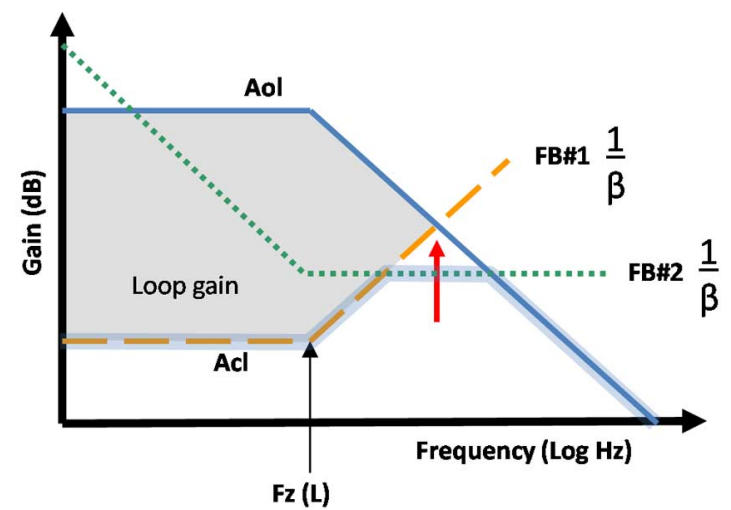

Fig. 7. Beta feedback analysis for multiple feedback paths. The dashed line is the feedback path formed by the inductor, Rf and Rs. The dotted line is the feedback path through the $\mathrm{Rd}$ and $\mathrm{Cf}$ high frequency shunt. The solid line represents the open-loop gain of the amplifier and the buffer stage. The arrow denotes a loop closure rate of $40 \mathrm{~dB}$ indicating an instability condition. $F z(L)$ denotes the zero created by including the active load in the feedback loop. The shaded line represents the closed-loop gain.

The first feedback loop (FB\#1 in Fig. 7) is through the inductor and the zero in the feedback path $\left(f_{z}(L)\right)$ is defined by the load inductance $\left(L_{l}\right)$ and parasitic resistance $\left(R_{l}\right)$ of the load as

$$
f_{z}(L)=\frac{R_{s}+R_{l}}{2 \pi f L_{l}} .
$$

If a second feedback path is not in place, the feedback loop and the amplifier's open-loop gain plot intersect with a closure rate of $40 \mathrm{~dB}$. A rate of closure of $40 \mathrm{~dB}$ corresponds to a phase shift of $180^{\circ}$ and oscillatory behavior. To compensate, a second feedback path with a feedback path zero (capacitor) is added to the circuit (FB\#2 in Fig. 7). The value of the capacitor is set below the feedback path of the load feedback path and the resistance is set to provide a rate of loop closure of $20 \mathrm{~dB}$ and at least $20 \mathrm{~dB}$ greater than the dc gain [59], [61]. The second feedback loop zero $\left(f_{z}(C)\right)$ is defined by the feedback capacitor $\left(C_{f}\right)$ and the feedback resistance $\left(R_{d}\right)$ as

$$
f_{z}(C)=\frac{1}{2 \pi f R_{d} C_{f}} .
$$

The rate of closure is a powerful technique, but for a complete analysis, it is important to check the open-loop phase shift throughout the gain bandwidth. This may be estimated by using hand plots or spreadsheets [62] but the use of Tian's method [63] for feedback-loop analysis more accurate and handily implemented in SPICE, such as the free switcherCAD from Linear technology [64].

\section{Output Stage Design Details}

There are a few implementation caveats to the floating load current source topology approach. Inductance of the sense resistor must be minimized to prevent peaking and oscillation. The value of $R_{s}$ impacts the loop gain and larger values improve the power bandwidth and the settling time from (18). In this topology, the entire current flows through the sense resistor and larger values will increase the voltage drive requirement. Unfortunately, monolithic operational amplifiers which have the necessary gain bandwidth and drive current in the ampere range 


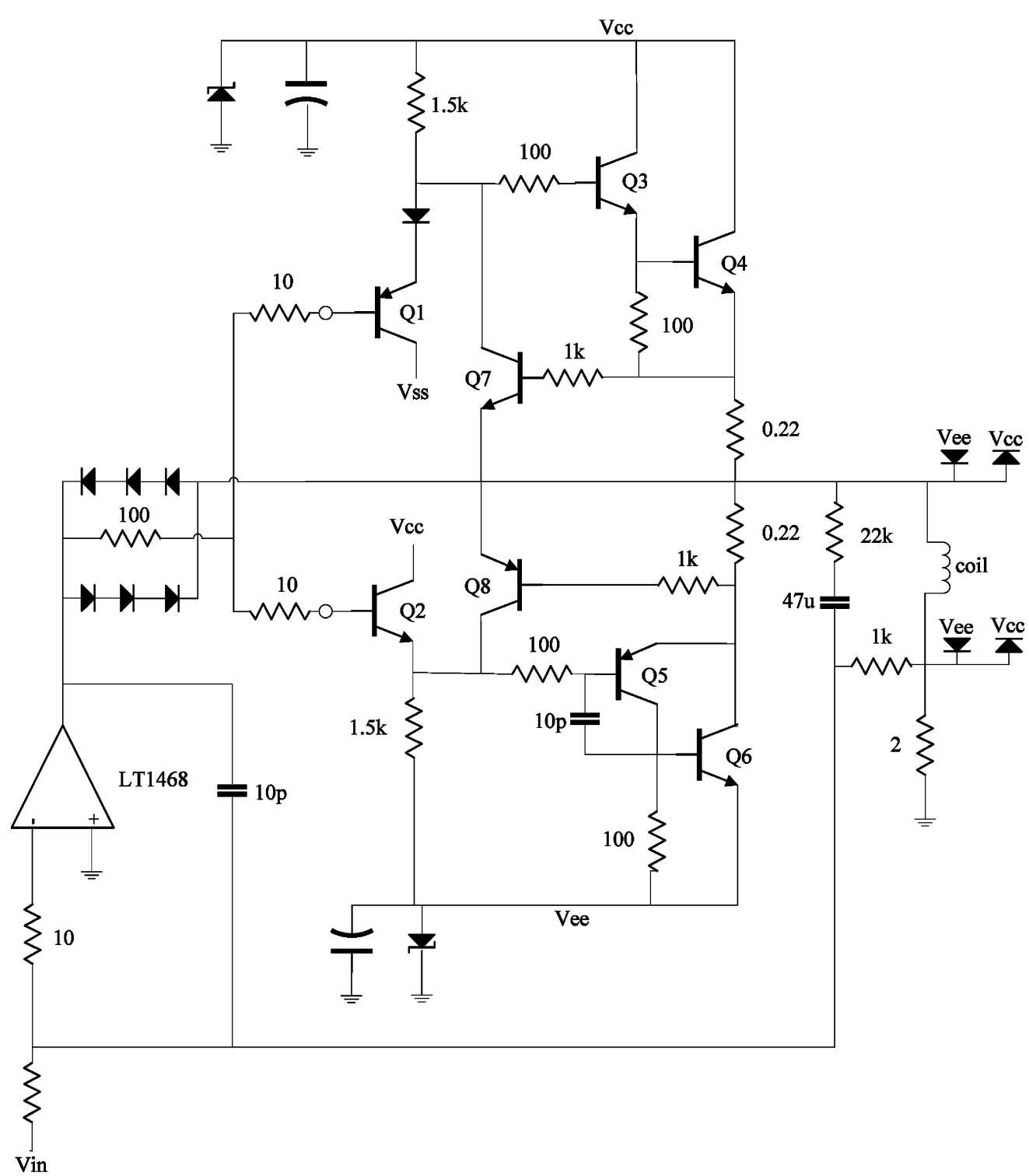

Fig. 8. Completed circuit. Transistors are Advanced Semiconductor Q1, $Q 8=2 N 2907 Q 2, Q 7=2 N 2222 Q 3=2 N 3866 Q 5=2 N 5160 Q 4, Q 6=$ $2 N 3632$, input diodes are $1 \mathrm{~N} 4148$, clamping diodes are BAV99, Zener diodes are 1.5KE16A, power-supply capacitors are low ESR electrolytic, $2200 \mu \mathrm{F}$. Small circles are optional single turn ferrite beads that can reduce high-frequency feedthrough. Vcc and Vee are $\pm 16 \mathrm{~V}$, respectively.

are not available. To meet this need, a composite amplifier with a commercially available current feedback amplifier (LT1468) and a discrete power-output stage (adapted from [65]) was developed. Current feedback amplifiers are ideal for this application as they provide moderate gains and a high unity gain bandwidth frequency with excellent drive capabilities.

For the power-output stage, bipolar power devices were selected because of their low output resistance, reduced drive requirements, and immunity to inductive kickback spikes which can destroy MOS transistors. However, it is generally difficult to find radio-frequency (RF) power PNP-type bipolar junction transistor (BJT) devices. The lack of high-speed PNP-type devices can be resolved by using composite devices [66].

Careful design is important because composite devices may develop local oscillations and have bandwidth limitations. Composite devices (Darlington or composite PNP-type) are required because the gain of power RF BJT devices is generally low. RF devices are used because the current booster stage must be significantly faster than the driving operational amplifier.
The speed requirement of the current booster stage is easily understood by referring to Fig. 7. If the current booster stage has a 3-dB roll off below the unity gain bandwidth of the amplifier, it will introduce and additional pole and invite oscillation. If the current feedback stage cannot respond to the output control signal from the amplifier, then the output will oscillate while the current booster continually tries to catch up to the feedback signal measured at the sense resistor. To prevent the addition of poles in the feedback loop, the midband gain of the power stage must extend past the unity gain frequency of the amplifier [65], [67].

Placing a reactive load inside the feedback loop requires particular attention when switching currents. Switched currents result in sharp flyback pulses from the inductive load. Flyback voltage pulses can damage components and create intermittent failures. The most effective way to dissipate flyback voltage pulses is to include discrete ultrafast recovery flyback diodes (Fig. 8 clamping diodes shown on the right). With the proper selection of components, the parasitic capacitance added to the output is negligible. 


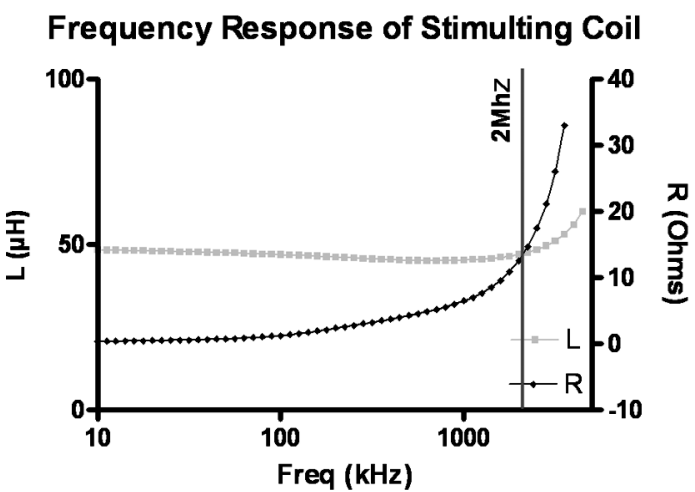

Fig. 9. Measurement results of small- signal coil impedance. Coil resistance is negligible below $200 \mathrm{~Hz}$, but becomes significant at $2 \mathrm{MHz}$.

Additional protection from flyback pulses is achieved by using unidirectional Zener diodes on the power-supply rails [68].

\section{EXPERIMENTAL RESULTS}

\section{A. Verification of Coil Through Operating Frequency}

One of the caveats to using a core as a flux concentrator is that the core must have an operational frequency above the highest frequency component of the driving waveform to reduce power losses due to the core. The frequency range for pulsed magnetic stimulation requires operation in the $200-\mathrm{kHz}-1-\mathrm{MHz}$ frequency. This mandates the use of ferrite cores as it exceeds the bulk of steel and Permalloy core performances. The cores tested in this system used cores from Fair-Rite Products Corporation (www.fair-rite.com) of material 77.

Performance up to $3 \mathrm{MHz}$ is possible with alternate materials, such as material 61. Custom machining for many shapes is available from several manufacturers, Elna Magnetics (www.elnamagnetics.com), for example.

We used a Hewlett-Packard 4192A impedance analyzer with a custom interface written in LabView (code available upon request from the corresponding author) to verify whethert the self-resonant frequency of the coil was above the operating frequency of the circuit. Alternately, the equivalent circuit analysis built in to the Hewlett-Packard/Agilent 4194A/4294A could be used to determine resonant frequency and equivalent circuit parasitic values. In-circuit operation was confirmed by analyzing the Vin/Iout waveform phase shift and shown to have a roll off frequency above $1 \mathrm{MHz}$. In this manner, the low-current and pulsed high-current performance of the cores may be verified. These two techniques were used because the 4192A cannot source high currents and because instrumentation for the low-frequency, high-current inductance measurement is not typically available and would likely overheat the coil as these coils are designed for a pulsed circuit topology, not continuous operation.

\section{B. Verification of the Current Waveforms}

The entire system was tested with a range of inductances from $1 \mu \mathrm{H}-100 \mu \mathrm{H}$, with and without cores, to verify system stability. The test inductors ranged from milliohm to $1-\Omega$ parasitic resistances.

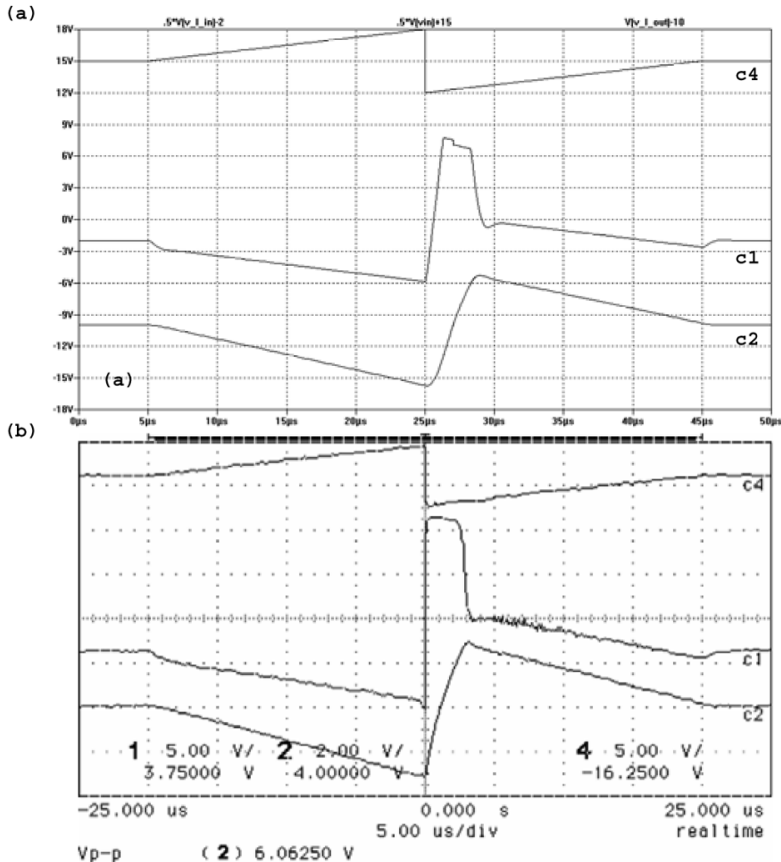

Fig. 10. Simulated and measured responses of a single ramp pulse for the reactive load driver circuit. (a) Waveforms from LT Spice simulation. (b) Measured response. C4 is the input waveform, $\mathrm{C} 1$ is the voltage at the load, and $\mathrm{C} 2$ is the voltage across the sense resistor.

Using hand manufacturing methods, it is reasonable to fabricate coils from 1-5 mm, which would have efficient penetrations depths from $100 \mu \mathrm{m}$ to $500 \mu \mathrm{m}$ according to analysis of the depth of penetration. The area of exposure will be limited by the sharpness of the peak and the dimensions of the coil. The range of inductances was chosen to correlate with the coils manufactured in lab. However, the circuit topology and the presented circuit are widely flexible. For a particular design, the stability of the system can be adjusted by adjusting the feedback pole and the dc gain. To obtain maximum frequency performance, increase the value of $R_{d}$ and, if necessary, reduce the value of $C_{f}$ until the circuit begins to ring with a square-wave voltage waveform input. The fastest transition possible will be a function of the power-supply voltage since $V=L(\mathrm{di} / \mathrm{dt})$. Step-response transitions are also limited by the amplifier slew rate and RLC network formed by the load parasitic resistance, the series inductance, and resistance of the supply leads, the switch resistance, and the bypass capacitance. While adding large bypass capacitors may prevent voltage droop, they will ultimately increase the switching time. Peak drive current performance is a primarily a function of the parasitic inductor resistance and the size of the sense resistor. Fig. 10 shows typical simulation and measurement results from a $20-\mu \mathrm{H}$ coil. For this case, the slew rate is measured at $1.3 \mathrm{~A} / \mu \mathrm{S}$. It is important to use precise probing techniques as the inductance of the probe can significantly influence the measured waveform for this circuit topology. Frequency and amplitude sweeps are shown in Figs. 11 and 12. As demonstrated in these two figures, the system performs as a V-I converter over a wide range of input voltage waveform amplitudes and shapes to drive inductive loads. 

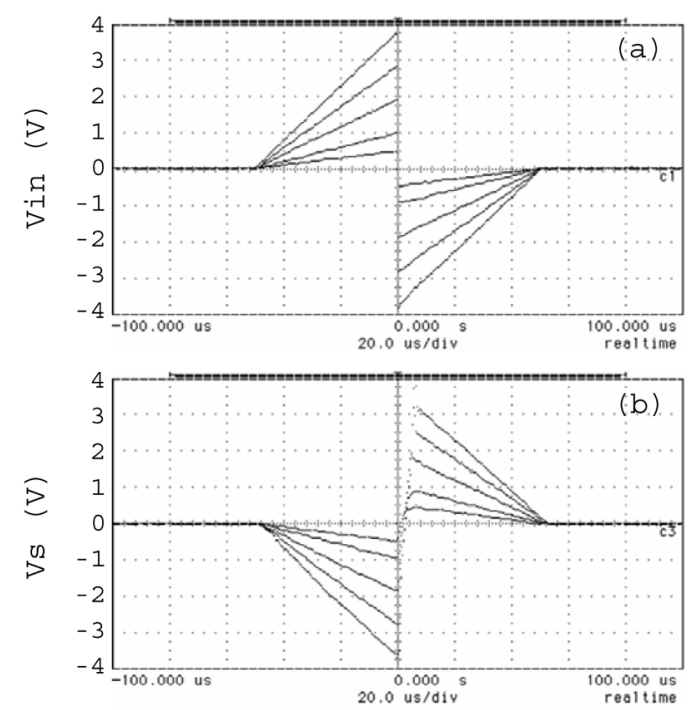

Fig. 11. Current output ramp response from an increasing voltage sawtooth input. Measured responses of ramp pulse swept in increasing input voltage for the reactive load driver circuit. (a) Input voltage waveforms. (b) Measured voltage across the $2-\Omega$ sense resistor, similar to $\mathrm{C} 2$ in Fig. 10 .

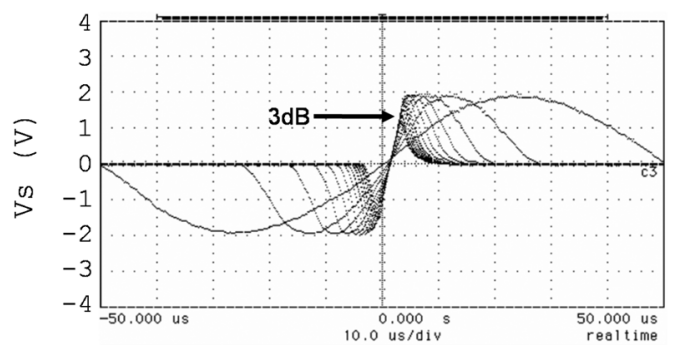

Fig. 12. Current output frequency response from a decreasing period sine-wave input. Sine-wave sweep of increasing frequency at half power measured at the sense resistor. The $3-\mathrm{dB}$ rolloff was measured at $129 \mathrm{kHz}$, indicated by the bold arrow.

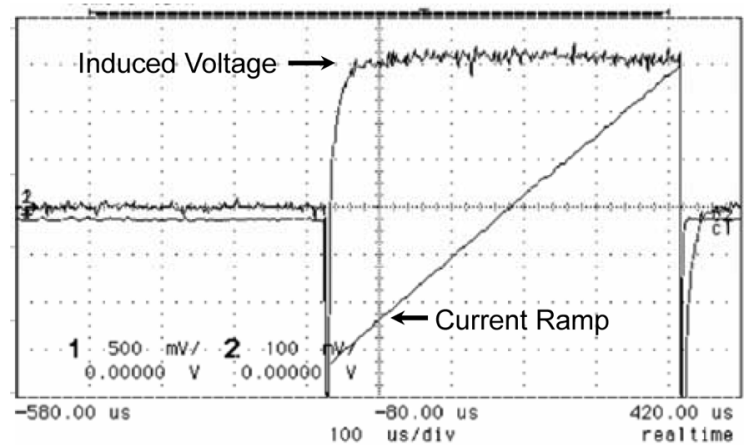

Fig. 13. Measurement of electric field generated in situ. The current ramp output of the amplifier clearly generates a nearly square voltage pulse. Ramp output waveform and measured response from the pickup coil area shown.

\section{Verification of the Electric Field}

To verify that the electric field was generated by the time-varying current pulse as predicted, a pickup coil was placed over the coil as the circuit generated single current sawtooth waveforms. The signal from the pickup coil was amplified by using an INA111 instrumentation op amp with a gain of 10X. The generated electric field should be a square wave, as was observed, shown in Fig. 13. While [69]-[71] discuss probe construction for measuring electric fields above and inside solenoid inductors, probe construction, accurate measurements, and proper alignment on the submillimeter scale proved unreasonable.

\section{CONCLUSION}

Recently, there has been an increase in interest in magnetic stimulation for use in peripheral, brain, and spinal nerve stimulation. Even though successful, repeatable magnetic stimulation was first reported in 1985 [72], little quantitative work has been performed to examine histological effects, observe intracellular responses, and map the response to the actual structures stimulated, such as heterogeneous neural structures.

With the development of the system presented, the opportunity to perform experiments with varying waveform, time sequencing, and distribution presents itself.

The flexibility of arbitrary current waveform generation comes at a cost. Creating a full-scale system based upon the linear current amplifier topology presented would be challenging and in all likelihood would require a modification of this approach. However, new power devices and pulse capacitors are continually under development [73]-[75] and supply the need for such devices in industrial applications. Switching strategies and circuit topologies can be tested in a smaller scale system before full-power devices become commercially available.

While uniform nerve fibers were highlighted in this paper, the proposed approach should also find utility in testing retina and dissociated cell in-vitro cultures commonly used in microelectrode array experiments (MEA) concerning synapse formation and network analysis. The circuit approach may find utility in other applications, such as magnetically-based cell sorting [76]. Scaling also will prove useful for studying small intact structures, such as brain slices and retina.

Simulation files, printed-circuit board layout files, parts lists, sourcing recommendations, and details of coil fabrication are available upon request by email to the authors.

\section{REFERENCES}

[1] N. Ishikawa, S. Suda, T. Sasaki, T. Yamanishi, H. Hosaka, K. Yasuda, and H. Ito, "Development of a non-invasive treatment system for urinary incontinence using a functional continuous magnetic stimulator (FCMS),” Med. Biol. Eng. Comput., vol. 36, pp. 704-10, 1998.

[2] J. Pujol, A. Pascual-Leone, C. Dolz, E. Delgado, J. L. Dolz, and J. Aldoma, "The effect of repetitive magnetic stimulation on localized musculoskeletal pain," Neuroreport, vol. 9, pp. 1745-1748, 1998.

[3] V. W. Lin and D. D. Cardenas, Spinal Cord Medicine: Principles and Practice. New York: Demos, 2003.

[4] A. T. Barker, "The history and basic principles of magnetic nerve stimulation," Electroencephalogr. Clin. Neurophysiol. Suppl., vol. 51, pp. 3-21, 1999.

[5] P. J. Basser and B. J. Roth, "New currents in electrical stimulation of excitable tissues," Аnnu. Rev. Biomed. Eng., vol. 2, pp. 377-397, 2000.

[6] L. A. Geddes, "History of magnetic stimulation of the nervous system," J. Clin. Neurophysiol., vol. 8, pp. 3-9, 1991.

[7] M. Hallett, "Transcranial magnetic stimulation and the human brain," Nature, vol. 406, pp. 147-150, 2000

[8] M. S. George, Z. Nahas, S. H. Lisanby, T. Schlaepfer, F. A. Kozel, and B. D. Greenberg, "Transcranial magnetic stimulation," Neurosurg. Clinics. North Amer., vol. 14, pp. 283-301, 2003.

[9] V. Walsh and A. Cowey, "Transcranial magnetic stimulation and cognitive neuroscience," J. Neurosci., vol. 19, pp. 5792-5801, 1999.

[10] A. Pascual-Leone, Handbook of Transcranial Magnetic Stimulation. New York: Arnold; Oxford University Press [distributor], 2002.

[11] H. A. C. Eaton, "The electric field induced in a spherical volume conductor by a magnetic coil," in, Proc. IEEE 12th Annu. Int. Conf. Engineering in Medicine and Biol. Soc., 1990, pp. 2247-2248. 
[12] M. Bencsik, R. Bowtell, and R. M. Bowley, "Electric fields induced in a spherical volume conductor by temporally varying magnetic field gradients," Phys. Med. Biol., pp. 557-576, 2002.

[13] P. Ravazzani, J. Ruohonen, F. Grandori, and G. Tognola, "Magnetic stimulation of the nervous system: Induced electric field in unbounded, semi-infinite, spherical, and cylindrical media," Ann. Biomed. Eng., vol. 24, pp. 606-616, 1996.

[14] B. J. Roth and P. J. Basser, "A model of the stimulation of a nerve fiber by electromagnetic induction," IEEE Trans. Biomed. Eng., vol. 37, no. 6, pp. 588-597, Jun. 1990.

[15] P. C. Miranda, L. Correia, R. Salvador, and P. J. Basser, "Tissue heterogeneity as a mechanism for localized neural stimulation by applied electric fields," Phys. Med. Biol., vol. 52, pp. 5603-5617, 2007.

[16] H. Ye, M. Cotic, and P. L. Carlen, "Transmembrane potential induced in a spherical cell model under low-frequency magnetic stimulation," J. Neural Eng., vol. 4, pp. 283-293, 2007.

[17] S. S. Nagarajan, D. M. Durand, and K. Hsuing-Hsu, "Mapping location of excitation during magnetic stimulation: Effects of coil position," Ann. Biomed. Eng., vol. 25, pp. 112-125, 1997.

[18] A. Rotem and E. Moses, "Magnetic stimulation of curved nerves," IEEE Trans. Biomed. Eng., vol. 53, no. 3, pp. 414-420, Mar. 2006.

[19] M. Kato, Electromagnetics in Biology. Tokyo, Japan: Springer, 2006.

[20] R.-R. Ji, T. E. Schlaepfer, C. D. Aizenman, C. M. Epstein, D. Qiu, J. C. Huang, and F. Rupp, "Repetitive transcranial magnetic stimulation activates specific regions in rat brain," in Proc. Nat. Academy Sci. United States America, Dec. 22, 1998, vol. 95, pp. 15635-15640.

[21] G. N. Li and D. Hoffman-Kim, "Tissue-engineered platforms of axon guidance," Tissue Eng. Part B: Rev., vol. 14, pp. 33-51, 2008.

[22] A. Rotem and E. Moses, "Magnetic stimulation of one-dimensional neuronal cultures," Biophys. J., 2008.

[23] E. Wasserman, C. M. Epstein, and U. Ziemann, The Oxford Handbook of Transcranial Stimulation. Oxford, U.K.: Oxford University Press, 2008.

[24] M. C. Ridding and J. C. Rothwell, "Is there a future for therapeutic use of transcranial magnetic stimulation?," Nat Rev Neurosci., vol. 8, pp. 559-567, 2007.

[25] B. J. Roth, "Mechanisms for electrical stimulation of excitable tissue," Crit. Rev. Biomed. Eng., vol. 22, pp. 253-305, 1994.

[26] F. Rattay, "Analysis of models for extracellular fiber stimulation," IEEE Trans. Biomed. Eng., vol. 36, pp. 676-682, 1989.

[27] B. J. Roth, "Mechanisms for electrical stimulation of excitable tissue," Crit. Rev. Biomed. Eng., vol. 22, pp. 253-305, 1994.

[28] K. P. Esselle and M. A. Stuchly, "Neural stimulation with magnetic fields: Analysis of induced electric fields," IEEE Trans. Biomed. Eng., vol. 39, no. 7, pp. 693-700, Jul. 1992.

[29] W. J. Havel, J. A. Nyenhuis, J. D. Bourland, K. S. Foster, L. A. Geddes, G. P. Graber, M. S. Waninger, and D. J. Schaefer, "Comparison of rectangular and damped sinusoidal $\mathrm{dB} / \mathrm{dt}$ waveforms in magnetic stimulation," IEEE Trans. Magn., vol. 33, no. 5, pt. 2, pp. 4269-4271, 1997.

[30] M. Sahin and Y. Tie, "Non-rectangular waveforms for neural stimulation with practical electrodes," J. Neural Eng., p. 227, 2007.

[31] A. T. Barker, C. W. Garnham, and I. L. Freeston, "Magnetic nerve stimulation: The effect of waveform on efficiency, determination of neural membrane time constants and the measurement of stimulator output," Electroencephal. Clin. Neurophys. Suppl., vol. 43, pp. 227-37, 1991.

[32] N. Accornero, G. Bini, G. L. Lenzi, and M. Manfredi, "Selective activation of peripheral-nerve fiber groups of different diameter by triangular shaped stimulus pulses," J. Physiol.-London, vol. 273, pp. 539-560, 1977.

[33] W. M. Grill, W. M. Grill, and J. T. Mortimer, "Stimulus waveforms for selective neural stimulation," IEEE Eng. Med. Biol. Mag., vol. 14, no. 4, pp. 375-385, Jul./Aug. 1995.

[34] A. Alkhateeb and R. P. Gaumond, "Excitation of frog sciatic nerve using pulsed magnetic fields effect of waveform variations," in Proc. IEEE 17th Annu. Conf. Engineering Medicine and Biology Soc., 1995, vol. 2, pp. 1119-1120.

[35] L. A. Geddes, "Accuracy limitations of chronaxie values," IEEE Trans. Biomed. Eng., vol. 51, no. 1, pp. 176-181, Jan. 2004.

[36] A. V. Peterchev, R. Jalinous, and S. H. Lisanby, "A transcranial magnetic stimulator inducing near-rectangular pulses with controllable pulse width (cTMS)," IEEE Trans. Biomed. Eng., vol. 55, no. 1, pp. 257-266, Jan. 2008.

[37] D. Perreault and S. Mogren, "Magnetic Stimulator Power and Control Circuit,” U.S. Patent 6551 233, Apr. 22, 2003, R.B. CArr Engineering, Onc.

[38] D. Prutchi and M. Norris, Design and Development of Medical Electronic Instrumentation: A Practical Perspective of the Design, Construction, and Test of Medical Devices. Hoboken, NJ: Wiley-Interscience, 2005.
[39] K. Davey and M. Riehl, "Designing transcranial magnetic stimulation systems," IEEE Trans. Magn., vol. 41, no. 3, pp. 1142-1148, Mar. 2005.

[40] B. H. Han, I. K. Chun, S. C. Lee, and S. Y. Lee, "Multichannel magnetic stimulation system design considering mutual couplings among the stimulation coils," IEEE Trans. Biomed. Eng., vol. 51, no. 5, pp. 812-817, May 2004.

[41] J. Ruohonen and R. J. Ilmoniemi, "Focusing and targeting of magnetic brain stimulation using multiple coils," Med. Biol. Eng. Comput., vol. 36, pp. 297-301, 1998.

[42] K. H. Hsu and D. M. Durand, "Prediction of neural excitation during magnetic stimulation usingpassive cable models," IEEE Trans. Biomed. Eng., vol. 47, no. 4, pp. 463-471, Apr. 2000.

[43] T. Kotnik, D. Miklavcic, and T. Slivnik, "Time course of transmembrane voltage induced by time-varying electric fields-a method for theoretical analysis and its application," Bioelectrochem. Bioenergetics, vol. 45, pp. 3-16, 1998.

[44] B. Han, S. Lee, J. Kim, and J. Yi, "Some technical aspects of magnetic stimulation coil design with the ferromagnetic effect," Med. Biol. Eng. Comput., vol. 41, pp. 516-518, 2003.

[45] K. Davey and C. M. Epstein, "Magnetic stimulation coil and circuit design," IEEE Trans. Biomed. Eng., vol. 47, no. 11, pp. 1493-1499, Nov. 2000.

[46] B. H. Han, S. Y. Lee, J. H. Kim, and J. H. Yi, "Some technical aspects of magnetic stimulation coil design with the ferromagnetic effect," Med. Biol. Eng. Comput., vol. 41, pp. 516-518, 2003.

[47] K. Davey, L. Luo, and D. A. Ross, "Toward functional magnetic stimulation (FMS) theory and experiment,", IEEE Trans. Biomed. Eng., vol. 41, no. 11, pp. 1024-1030, Nov. 1994.

[48] A. van den Bossche, Inductors and Transformers for Power Electronics. Boca Raton, FL: CRC, 2005.

[49] A. Van den Bossche, V. Valchev, and T. Filchev, "Improved approximation for fringing permeances in gapped inductors," in Proc. Conf. Rec. 37th Industry Applications Soc. Annu. Meet., 2002, vol. 2, pp. 932-938

[50] C. W. T. McLyman, W. T. McLyman, and M. McLyman, Transformer and Inductor Design Handbook. Boca Raton, FL: CRC Press, 2004.

[51] K. P. Esselle and M. A. Stuchly, "Cylindrical tissue model for magnetic field stimulation of neurons: Effects of coil geometry," IEEE Trans. Biomed. Eng., vol. 42, no. 9, pp. 934-941, Sep. 1995.

[52] A. Alkhateeb, "Excitation of frog sciatic nerve using magnetic induction," Ph.D. dissertation, Pennsylvania State Univ., University Park, PA, 1997.

[53] J. D. Sommers and R. P. Gaumond, "Spacing analysis of a focal magnetic stimulating coil," in Proc. IEEE 23rd Northeast Bioengineering Conf., 1997, pp. 42-43.

[54] L. Zeng, "An electrode array for reversing the recrutimnet order of peripheral nerve stimulation," Ph.D. dissertation, Case Western Reserve Univ., Cleveland, OH, 2004.

[55] B. Mulloney, N. Tschuluun, and W. M. Hall, "Architectonics of crayfish ganglia," Microscopy Res. Technique, vol. 60, pp. 253-265, 2003.

[56] R. M. Glantz and T. Viancour, "Integrative properties of crayfish medial giant neuron: Steady-state model," J Neurophysiol., vol. 50, pp. 1122-1142, Nov. 1983.

[57] L. Zeng and M. D. Dominique, "Extracellular voltage profile for reversing the recruitment order of peripheral nerve stimulation: A simulation study," J. Neural Eng., p. 202, 2004.

[58] S. Franco, Design With Operational Amplifiers and Analog Integrated Circuits, 3rd ed. New York: McGraw-Hill, 2002.

[59] J. Steele and T. Green, "Tame those versatile current source circuits," Elect. Des, pp. 61-72, 1992.

[60] A. S. S. a. K. C. Smith, Microelectronic Circuits, 4th ed. Oxford, U.K.: Oxford University Press, 1998.

[61] Apex Technology, "Stability for power amplifiers." Appl. Note 19 (AN19UREVC), Nov. 2004. [Online]. Available: http://apex.cirrus. com/en/pubs/appNote/Apex_AN19U_C.pdf

[62] Appl. Note 38. "Loop stability with reactive loads" A. Microtechnology.

[63] M. Tian, V. Visvanathan, J. Hantgan, and K. Kundert, "Striving for small-signal stability," IEEE Circuits Devices Mag., vol. 17, no. 1, pp. 31-41, Jan. 2001.

[64] M. Engelhardt, Simulationsprogramm LT_SPICE (SwitcherCAD III).

[65] J. Williams, "High speed amplifier techniques," 1991, vol. 47, Appl. Note, Linear Technology Corp..

[66] P. R. Gray, Analysis and Design of Analog Integrated Circuits, 4th ed. New York: Wiley, 2001

[67] J. Williams, "Power gain stages for monolithic amplifiers," 18, 1986, Appl. Note, Linear Technology Corp. 
[68] J. Steele, "Protect those expensive power op amps," Electronic Design, Jan. 1991.

[69] R. L. Stenzel, "A new probe for measuring small electric fields in plasmas," Rev. Scientif. Instrum., vol. 62, pp. 130-130, 1991.

[70] P. M. Glover and R. Bowtell, "Measurement of electric fields due to time-varying magnetic field gradients using dipole probes," Phys. Med. Biol., vol. 52, pp. 5119-5130, 2007.

[71] L. E. E. Soonchil, L. E. E. Yongkwan, and Y. U. Insuk, "Electric field in solenoids," Jpn. J. Appl. Phys., vol. 44, pp. 5244-5248, 2005.

[72] A. T. Barker, R. Jalinous, and I. L. Freeston, "Non-invasive magnetic stimulation of human motor cortex," Lancet, vol. 1, pp. 1106-7, 1985.

[73] B. Travis, "IGBTs and MOSFETs vie for applications," EDN, vol. 44, pp. 77-88, 1999.

[74] A. Burke, "Ultracapacitors: Why, how, and where is the technology," J. Power Sources, vol. 91, pp. 37-50, 2000.

[75] K Slenes, P. Winsor, T. Scholz, M. Hudis, T. Inc, and N. M. Albuquerque, "Pulse power capability of high energy density capacitors based on a new dielectric material," IEEE Trans. Magn., vol. 37, no. 1, pt. 1, pp. 324-327, Jan. 2001.

[76] M. Frenea-Robin, H. Chetouani, N. Haddour, H. Rostaing, J. Laforet, and G. Reyne, "Contactless diamagnetic trapping of living cells onto a micromagnet array," in Proc. IEEE Annu. Int. Conf. Engineering Medicine and Biology Soc., , 2008, pp. 3360-3363.

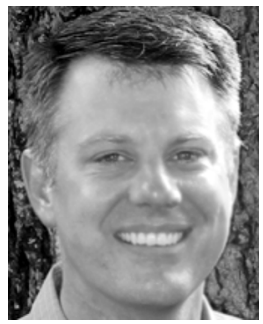

Eric Basham (S'09) received the B.S. degree in biotechnology from Cook College, Rutgers University, Piscataway, NJ, in 1997, the M.S.E.E. degree from San Jose State University, San Jose, CA, in 2006, and is currently pursuing the Ph.D. degree in electrical engineering from the University of California, Santa Cruz.

He has been a Technician with Marigenetics and HP Labs and a Summer Research Intern with Rutgers University, Aclara, and Intel. His research interests include transistor design for sensing applications, fundamental aspects of neural interfacing, field-programmable analog arrays, and biologically inspired computing.

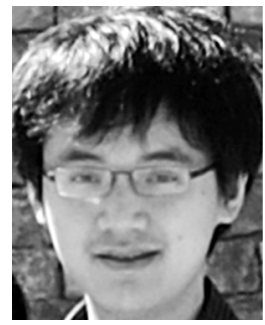

Zhi Yang was born on November 29, 1981, in China. $\mathrm{He}$ received the B.S. degree in electrical engineering from Zhejiang University, Zhejiang, China, in 2004 and the M.S. degree in electrical engineering from the University of California at Santa Cruz in 2007, where he is currently pursuing the Ph.D. degree.

Since 2005, he has been with the University of California at Santa Cruz. His research interests are mathematical modeling, analog circuit design, data analysis, and neuroprosthetic devices.

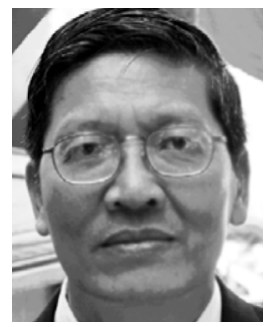

Wentai Liu (S'78-M'81-SM'93) received the B.S. degree from National Chiao-Tung University, Taiwan, the M.S. degree from National Taiwan University, and the Ph.D. degree from the University of Michigan, Ann Arbor.

In 1983, he joined North Carolina State University, Raleigh, where he held the Alcoa Chair Professorship in the Department of Electrical and Computer Engineering. Since 2003, he has been a Professor in the Electrical Engineering Department at the University of California at Santa Cruz, where he is Campus Director of the National Science Foundation Engineering Research Center on Biomimetic Microelectronic Systems. His research interests include visual prosthesis, implantable electronics, high-speed transceiver design (wired and wireless), molecular electronics, microelectronic sensors, timing/clock optimization, on-chip interconnects, and computer vision/image processing. Since its early stages, he has been leading the engineering efforts of the retinal prosthesis to restore vision, finally leading to successful preliminary implant tests in blind patients. He has published more than 180 technical papers and is a co-author of Wave Pipelining: Theory and CMOS Implementation (Kluwer, 1994) and Emerging Technologies: Designing Low Power Digital Systems (IEEE Press, 1996).

Dr. Liu received an IEEE Outstanding Paper Award, Alcoa Foundation's Distinguished Engineering Research Award, and the Outstanding Alumnus Award from National Chiao-Tung University. He is a University System of Taiwan Master Lecturer and Chair Professor of National Chiao-Tung University. 